\title{
adesso: Über das Entwickeln von Software und IT-Unternehmen
}

\author{
Rüdiger Striemer ${ }^{1}$ Andreas von Hayn ${ }^{1}$ \\ Angenommen: 11. September 2021 / Online publiziert: 20. Oktober 2021 \\ (c) Der/die Autor(en) 2021
}

\section{Zusammenfassung}

adesso ist heute ein internationaler IT-Dienstleister mit über 5000 Mitarbeitenden und Niederlassungen in ganz Deutschland sowie zahlreichen europäischen Ländern. Vor gut 25 Jahren war adesso die Idee von zwei Personen. Die Gründer wollten das wirtschaftliche Potenzial von IT für Unternehmen einfacher nutzbar machen. Von der Ursprungsidee - Prozesse in Softwareentwicklungsprojekten möglich exakt zu modellieren - blieb nicht viel übrig. Was blieb, ist die Leidenschaft für Software. Und ein großer Pragmatismus im Umgang mit Entscheidungen und Problemen. Der Erfolg von Java war entscheidend für den Erfolg des IT-Dienstleisters. Das erste Java-Projekt setzte adesso für Versicherungen um. Auf die Versicherungsindustrie folgten weitere Branchen. Aus reinen Softwareentwicklungsaufträgen wurden häufiger Projekte, in denen Beratung bei der IT-Strategie eine Rolle spielte. Aber im Kern ging es immer darum, wirtschaftliches Potenzial, ausgewiesene Fachleute und die richtigen Technologien zusammenzubringen. Eine der Herausforderungen für adesso ist das Managen des Wachstums. So ist das Finden geeigneter Expertinnen und Experten ein zentrales Thema. Es gilt, Menschen an Bord zu holen, die Fachwissen mitbringen und den adesso-Stil zu schätzen wissen.

„Business. People. Technology“: Mit diesem Dreiklang beschreibt der IT-Dienstleister adesso seine Tätigkeit. Hinter den 3 Begriffen verbergen sich 25 Jahre mit Kunden und Projekten, Wachstum und Einbrüchen. Im Laufe dieser aus heutiger Sicht langen Zeit unternahmen die Verantwortlichen mehrere Anläufe, den Claim anzupassen. Immer wieder kehrten sie zu „Business. People. Technology“ zurück. Kompakter ließen sich Selbstverständnis, Anspruch und Organisation nicht auf den Punkt bringen: Im Zentrum stehen die Menschen. Sie bringen Fachwissen, Engagement und die passenden Werkzeuge mit. So sorgen sie dafür, dass aus Technologiepotenzialen echtes Geschäft für Kunden entsteht. Diese Leidenschaft eint 2021 über 5000 Mitarbeitende in Europa. Sie war bereits 1997 die Grundlage für zwei Personen - Volker Gruhn und Rainer Rudolf - die in Dortmund ihr Projekt ,adesso - the process company“ aus der Taufe hoben. Abgesehen von diesem Grundverständnis blieb allerdings nicht viel übrig von den ersten Ideen. Die beiden Gründer entwickelten das Unternehmen - und das

Rüdiger Striemer

striemer@adesso.de

Andreas von Hayn

andreas.von-hayn@adesso.de

1 adesso SE Dortmund, Adessoplatz 1, 44269 Dortmund, Deutschland
Unternehmen entwickelte sich - in eine andere Richtung als geplant (Abb. 1).

\section{Am Anfang alles andere als agil}

Wie können Softwareentwicklungsabteilungen in großen Unternehmen besser arbeiten? Diese Frage stellten sich Gruhn und Rudolf Mitte der 1990er-Jahre. Aus eigenen Forschungs- und Promotionsaktivitäten brachten sie Modellierungsmethoden für Vorgehensmodelle auf Basis von Petri-Netzen mit. Mithilfe solcher Methoden wollten sie die Prozesse in der Softwareentwicklung exakt modellieren, formal beschreiben und besser planen. Das Ziel: Das Risiko in größeren Softwareentwicklungsprojekten reduzieren. Vom agilen Geist der Softwareentwicklung war hier noch nichts zu spüren. Das agile Manifest, das die Arbeit in Projekten später nachhaltig verändern sollte, war noch nicht einmal geschrieben.

Prozessmodellierung war also das Geschäftsmodell der „process company“. Das Start-up gewann erste Kunden aus der Telekommunikationsbranche. Die beiden Gründer schauten sich Abläufe an, modellierten, organisierten und optimierten. Die Kunden freuten sich über die Effizienzgewinne und vor allem: Planungssicherheit. Und wie es mit zufriedenen Kunden in der Regel ist: Sie wollten mehr. Statt die Prozesse nur zu gestalten, sollte adesso bei der 
Abb. 1 Entwicklung von adesso

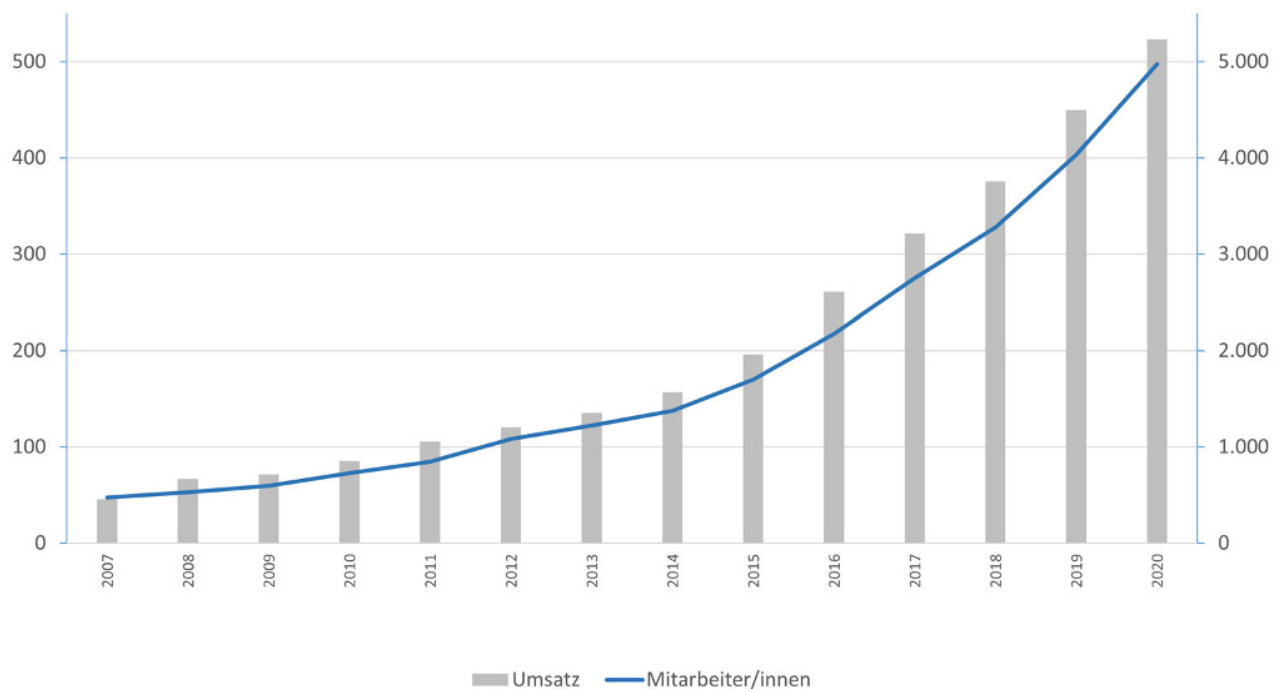

Softwareentwicklung aktiv unterstützen und Verantwortung übernehmen. Eine Geschäftsgelegenheit tat sich auf. Und mit Java stand eine vielversprechende neue Technologie vor der Tür, die zu den Kunden passte - jedenfalls sah es danach aus, wissen konnte man das damals nicht.

Auf technischer Ebene brachte Java alles mit, was das Herz der Softwareexpertinnen und -experten höherschlagen lässt: Plattformunabhängigkeit, Objektorientierung, eine Laufzeitumgebung, die einen Compiler überflüssig macht. All das war Ende der 1990er-Jahre eine Revolution für die Softwareentwicklung. Aber eine Frage war noch nicht geklärt: Eignet sich Java als Grundlage für ernsthafte Geschäftsanwendungen? Konnten die Fachleute von adesso auf dieser Basis zum Beispiel eine Bestandsführungssoftware für eine Versicherung entwickeln? Eine Anwendung, mit der Tausende von Sachbearbeitern täglich Kunden erfassen, Verträge verwalten, Vorgänge bearbeiten würden?

adesso sah in der Antwort auf diese Frage eine einmalige Chance für das junge Unternehmen: Wenn das mit Java funktioniert, eröffnet sich ein großer Markt. Dann warten da draußen lauter Unternehmen, die ihre Software auf eine zeitgemäße Grundlage heben wollen. Ein Plan B - für den Fall, dass Java nicht geeignet wäre - fiel der Euphorie über die technischen Möglichkeiten zum Opfer.

Mit der damaligen Zürich Agripina Versicherung fand adesso einen Kunden, der diese Herausforderung ähnlich risikofreudig anging. Gruhn und Rudolf heuerten aus ihren Kreisen eine Reihe Softwarefachleute an, die sich auf das Thema stürzten. Am Ende entwickelten die Versicherung und der IT-Dienstleister im Jahr 2000 die erste auf dem deutschen Markt verfügbare Bestandsführungssoftware auf Java-Basis. Rückblickend war dies die eigentliche Geburtsstunde von adesso. In diesem Projekt schufen die Beteilig- ten vieles von dem, was das Unternehmen das kommende Vierteljahrhundert prägte: Die Leidenschaft für Technologie. Der Wille, aus Technologie Geschäft für Kunden zu schaffen. Das Bewusstsein, dass das Verständnis des Kunden, seiner Prozesse und seiner Branche der Schlüssel für erfolgreiche IT-Projekte ist. Und eine gewisse Hemdsärmeligkeit im Umgang mit Prozessen und Formalismen. „Da setzen wir auf gesunden Menschenverstand“ entwickelte sich zum geflügelten Wort innerhalb von adesso.

Auf die Zürich Versicherung folgten weitere Versicherungen. Auf die Versicherungsindustrie folgten weitere Branchen. Aus reinen Softwareentwicklungsaufträgen wurden häufiger Projekte, in denen Beratung bei der ITStrategie eine Rolle spielte. Aber im Kern ging (und geht es bis heute) darum, Business, People und Technology zusammenzubringen.

\section{Tragfähige Strukturen statt eines engen Korsetts}

Parallel zu den zunehmend größeren und vielfältigeren Projekten entwickelte sich adesso organisatorisch weiter. In der anarchischen ersten Phase gab es kaum Blaupausen oder Prozesse. Jeder übernahm, was gerade anfiel - ob Personalarbeit, Vertrieb oder Projektorganisation. Im Zentrum versuchte das Gründerteam, so gut es ging, die Fäden zusammenzuhalten. Wenn die ganze Belegschaft in einen mittelgroßen Besprechungsraum passt, lässt sich auf diese Art vieles managen. Aber mehr Projekte und mehr Mitarbeitende erforderten eben dann doch irgendwann mehr Struktur. adesso etablierte sogenannte Lines of Business (LoB). Diese wurden nach Branchen (Versicherung, Banking etc.) 
Abb. 2 Seminarreise: Regelmäßig treffen sich die adessi irgendwo in Europa für ein verlängertes Wochenende zwischen Arbeit und Party

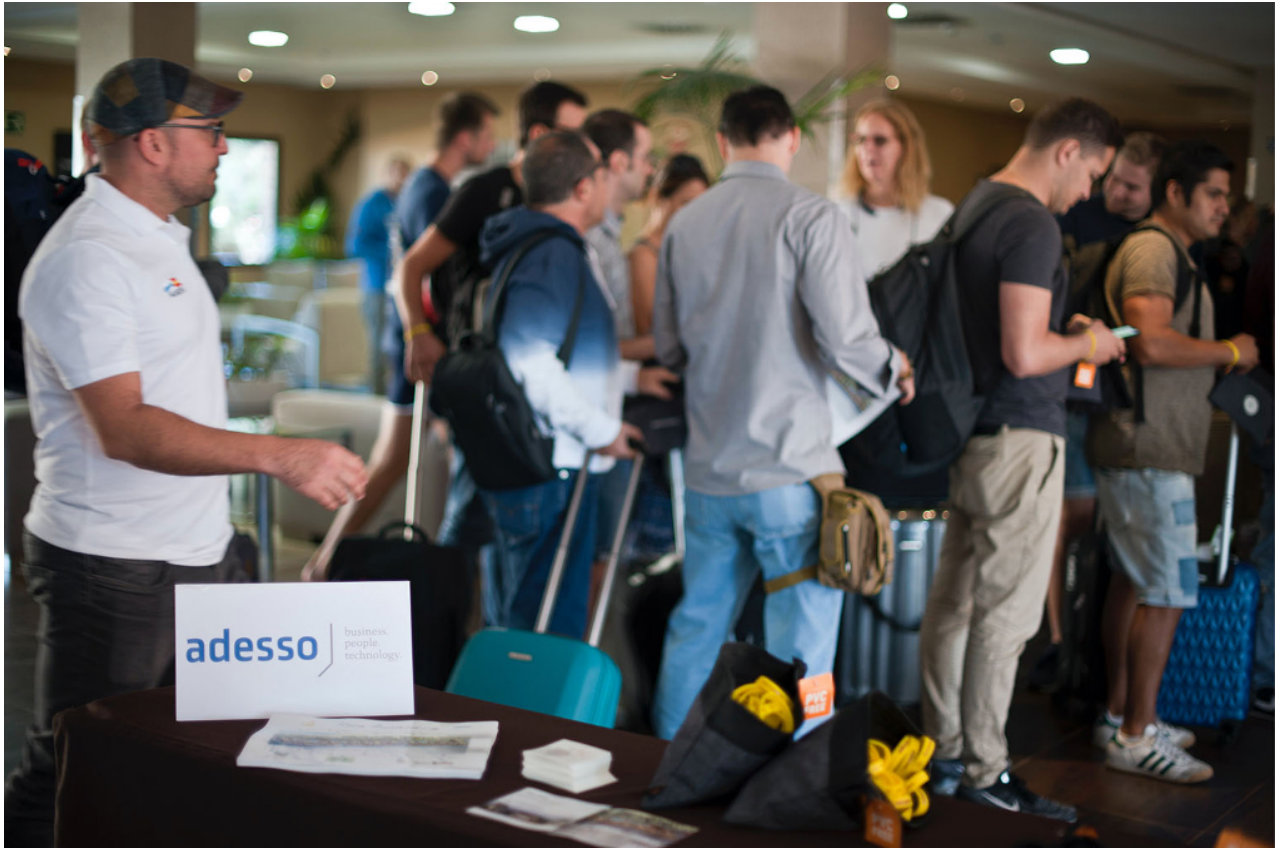

ausgerichtet. Die Idee der LoBs: adesso sollte jeweils in einem kleineren Maßstab, mit klarem Industriefokus, nachgebaut werden. In den Einheiten bündeln sich bis heute, mit großem Spielraum für Entscheidungen ausgestattet, Branchenkenntnisse und IT-Know-how. Sie agieren so autonom wie möglich, aber mit klaren Vorgaben was Lieferversprechen, Qualität und Service angeht. Mit dem Modell der LoBs gelang es den adesso-Entscheidern, Strukturen zu etablieren, in denen die dynamische Entwicklung der Anfangsjahre weiterlebte.

Eine LoB spielt in diesem Zusammenhang eine besondere Rolle: Cross Industries (oder kurz CI). Hier verortet adesso alle Kunden, Projekte und Technologien, die sich noch keiner ausgeprägten Branche zuordnen lassen. Cross Industries entwickelte sich zu einem Testlabor für das Unternehmen. Verantwortliche sammeln erste Erfahrungen mit neuen Branchen, Werkzeugen oder Plattformen. Diese Line of Business ist also quasi die Keimzelle für zukünftige Geschäftsbereiche: Erreicht ein Thema innerhalb von CI eine gewisse Größe, wird eine neue LoB ausgegründet. Dabei ist CI keine abgetrennte Organisationseinheit, die irgendwo in einem hippen Berliner Loft vor sich hin werkelt, aber keine Geschäftsverantwortung hätte. Die Mitarbeiterinnen und Mitarbeiter leisten ihren Beitrag zum Unternehmensergebnis; sie sind eingebunden in Controllingprozesse und Erfolgsmessung.

In den LoBs entwickelte sich ein weiteres wirtschaftliches Standbein von adesso: das Produktgeschäft. Das klassische Geschäft des IT-Dienstleisters ist immer noch der Kern des Unternehmens. Aber für Versicherungen und Lotteriegesellschaften entwickelten die Fachleute stan- dardisierte Softwarelösungen, die inzwischen namhafte Kunden in aller Welt einsetzen. Der weitere Ausbau des Produktangebots ist ein wichtiger Punkt auf der Agenda des adesso-Managements.

Neben mehr Struktur war und ist Nähe zu Kunden und Mitarbeitenden ein Erfolgsfaktor. Nach Dortmund kamen Standorte in Köln, Berlin, Frankfurt und München - aktuell in 25 deutschen Städten - hinzu. Bald war Nähe aber auch ein internationales Thema. Einerseits, um deutsche Kunden bei ihren länderübergreifenden Aktivitäten zu unterstützen. Andererseits, um lokales Geschäft vor Ort aufzubauen. Und auch die Möglichkeit, mit internen Kräften Nearshoring-Projekte auf- und umzusetzen, spielte in den Überlegungen eine Rolle. Mit adesso Schweiz und adesso Austria starteten die Aktivitäten zunächst im deutschsprachigen Ausland. Mit der Türkei dann erstmalig außerhalb der eigenen Sprachregion. Inzwischen ist adesso in $10 \mathrm{eu}-$ ropäischen Ländern vertreten. Der letzte Neuzugang in der adesso-Familie ist mit adesso Nordics ein Standort in Finnland. Die ersten Schritte in den asiatischen Markt bereitet das Management aktuell vor. In jeder neuen Ländergesellschaft kopieren die Verantwortlichen, angepasst an die lokalen Besonderheiten, das Wachstumsmodell der adessoGründungszeit.

Alles Planen und alles Strukturieren schützen aber nicht vor dem Moment, der für jeden IT-Dienstleister unweigerlich kommt: die erste Havarie. adesso machte diese Erfahrung im Jahr 2002, ausgerechnet mitten im Platzen der Dotcom-Blase, also in einer ohnehin schwierigen Zeit für einen IT-Dienstleister. Ein Projekt lief komplett aus dem Ruder, das Team riss Budgets und Termine. Dieses eine Projekt 
entwickelte sich zu einer Gefahr für das ganze Unternehmen. Die Aufregung war entsprechend groß, die Erfahrung im Umgang mit solchen Situationen allerdings klein. Verständnisvolle Kunden und engagierte Mitarbeitende lösten die akuten Schwierigkeiten - aber es war knapp. Den Verantwortlichen war klar, dass adesso sich besser wappnen muss, um nicht erneut blind in eine ähnliche Lage zu geraten. Eine Konsequenz: Das Unternehmen führte Methoden wie die Earned-Value-Analyse ein. Solche Methoden wurden im Laufe der Jahre verfeinert und ergänzt, ohne die wesentliche Qualität der Organisation zu beeinträchtigen: Freiraum für kluge Ideen. Das detaillierte Bild über die wirtschaftliche Performance einzelner Projekte sorgte für mehr Transparenz und bessere Entscheidungen. Heute ist adesso in der Lage, potenzielle Havarien spätestens nach einem Drittel der Projektlaufzeit zu erkennen. Was bis dahin unauffällig ist, wird auch nicht mehr zum Problem das lässt sich mit hoher Signifikanz aus über 400.000 Datenpunkten vergangener Projekte ableiten.

Hinzu kam das Platzen der Dotcom-Blase, das eine Reihe junger Hightech-Unternehmen mit in den Abgrund riss. Der Börsengang von adesso lag noch einige Jahre in der Zukunft, das Unternehmen geriet nicht unmittelbar in den finanziellen Strudel. Aber einige Kunden hatte es erwischt. Projekte wurden eingestellt, Zahlungen fielen aus. Die Unsicherheit über die Entwicklung war groß. Das Management stand vor der Entscheidung: Mitarbeitende entlassen, um Kosten zu senken. Oder darauf bauen, dass dies nur eine Delle in einer eigentlich intakten Geschichte ist. Das Vertrauen in die eigene Standhaftigkeit gewann. Mit einer Mischung aus „Gürtel enger schnallen“ und „Vertriebsfeuer aus allen Rohren“"gelang dem Unternehmen die Wende. 20 Jahre später wirkt diese Phase noch nach. Denn zahlreiche Kolleginnen und Kollegen von damals sind heute noch Teil des Teams - und wissen, wie wichtig das solide Umgehen mit Geld ist.

\section{Mehr als nur Kennzahlen}

Wachsen bedeutet aber nicht nur größere Zahlen, also mehr Mitarbeitende, mehr Projekte, mehr Standorte. Wachsen bedeutet vor allem, Werte weiterzureichen. Werte, die sich nicht auf dem Niveau von Kalendersprüchen bewegen, mit denen sich Eingangsbereiche und Besprechungsräume zieren lassen. Sondern die das tagtägliche Handeln prägen. Dazu gehört bei adesso neben dem bereits erwähnten gesunden Menschenverstand vor allem Respekt. Vor Mitarbeitenden, Kunden und den Herausforderungen des Alltags. Aber auch das Primat des Vertriebs, eine westfälische Grundhaltung des „Fertigmachens“ und - Neugier. Wer bei adesso arbei- tet, will wissen, wie das geht, egal, was „das“ im Einzelfall ist. Wenn zwei Gründer den ersten, zweiten oder dritten Mitarbeitenden an Bord holen, drängt sich das Thema der Wertevermittlung noch nicht auf. Sie suchen sich dann halt Personen aus, die zu ihren Vorstellungen passen. Später wird es schwieriger, denn gleichzeitig muss das Unternehmen neue Technologien adaptieren, immer mehr fachliche Kontexte bearbeiten können und - eben wachsen.

Noch 2021 begrüßt adesso Kollegin oder Kollege Nummer 6000. Das Personalwachstum erfordert nicht nur eingespielte Prozesse rund um alle Aspekte des Recruitings - von der Stellenanzeige bis zum Onboarding. Das erfordert eine Menge Fingerspitzengefühl bei den Beteiligten. Das Finden der passenden neuen Kolleginnen und Kollegen ist seit 25 Jahren der Erfolgsfaktor von adesso. Passend bedeutet einerseits das nötige Fachwissen, das sich meist auf Basis von Abschlüssen oder Berufserfahrung demonstrieren lässt. Passend bedeutet aber auch - und dies ist genauso wichtig - Menschen zu finden, die den adesso-Stil zu schätzen wissen.

Nach fast 25 Jahren kontinuierlichem Umsatz- und Ergebniswachstums, mehrfacher Auszeichnung als Deutschlands bestem Arbeitgeber und zunehmendem internationalem Erfolg scheint all das gelungen zu sein. Man könnte einer neuen Gründergeneration in der Informatik also mitgeben, ähnliche Werte und Prinzipien zu beherzigen.

Besser wäre, man lässt sie einfach machen. So wie uns damals (Abb. 2).

Open Access Dieser Artikel wird unter der Creative Commons Namensnennung 4.0 International Lizenz veröffentlicht, welche die Nutzung, Vervielfältigung, Bearbeitung, Verbreitung und Wiedergabe in jeglichem Medium und Format erlaubt, sofern Sie den/die ursprünglichen Autor(en) und die Quelle ordnungsgemäß nennen, einen Link zur Creative Commons Lizenz beifügen und angeben, ob Änderungen vorgenommen wurden.

Die in diesem Artikel enthaltenen Bilder und sonstiges Drittmaterial unterliegen ebenfalls der genannten Creative Commons Lizenz, sofern sich aus der Abbildungslegende nichts anderes ergibt. Sofern das betreffende Material nicht unter der genannten Creative Commons Lizenz steht und die betreffende Handlung nicht nach gesetzlichen Vorschriften erlaubt ist, ist für die oben aufgeführten Weiterverwendungen des Materials die Einwilligung des jeweiligen Rechteinhabers einzuholen.

Weitere Details zur Lizenz entnehmen Sie bitte der Lizenzinformation auf http://creativecommons.org/licenses/by/4.0/deed.de. 


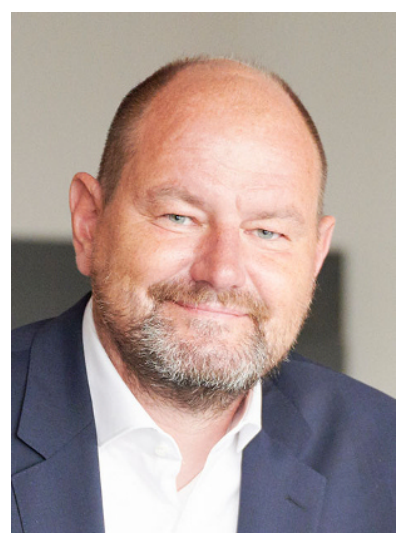

Rüdiger Striemer studierte Wirtschafts- und Sozialwissenschaften an der Universität Dortmund und hat an der Technischen Universität Berlin in Informatik promoviert. Nach seiner Tätigkeit als wissenschaftlicher Mitarbeiter am Fraunhofer-Institut für Software- und Systemtechnik kam er zur adesso $\mathrm{SE}$, wo er als langjähriger CoVorsitzender des Vorstands für die Softwareentwicklung verantwortlich war. Rüdiger Striemer leitet den Ausbau des internationalen Geschäfts der adesso SE und ist Professor für Wirtschaftsinformatik

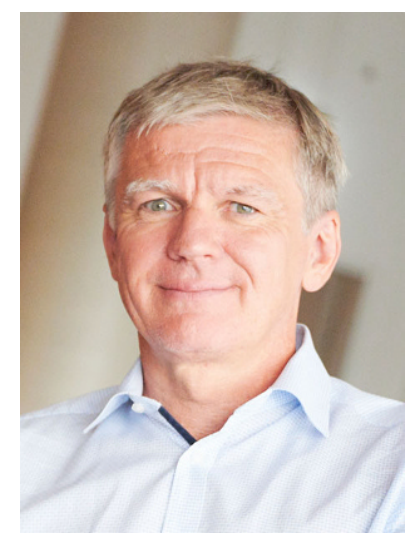

Andreas von Hayn studierte an der Ruhr-Universität Bochum Wirtschaftswissenschaft mit den Schwerpunkten Marketing und Sozialpsychologie. Nach Stationen im SAP- und Microsoft-Partnerumfeld ist er seit 2012 im Bereich Corporate Communications der adesso SE aktiv. Hier organisiert er unter anderem Kommunikationskampagnen rund um Themen wie Digitale Transformation oder künstliche Intelligenz. PR-/Marketing-Manager.

an der Technischen Hochschule Wildau. 